\title{
Research Progress on Soil Erosion and Socioeconomic Correlation
}

\author{
Yan Sun ${ }^{1,3}$, Junbo Xiao ${ }^{1,3}$, Yiyuan Zhang ${ }^{2}$, Wenhao Lai ${ }^{1,3}$, Min Wei ${ }^{1,3}$ and Junming Wang ${ }^{1,3^{*}}$ \\ ${ }^{1}$ Guangxi Hydraulic Research Institute, Nanning, Guangxi, 530023, China \\ ${ }^{2}$ Nanning Reconnaissance \& Designing Institute of Pearl River Water Resources Commission in Guangxi, Nanning, Guangxi, 530007, \\ China \\ ${ }^{3}$ Guangxi Key Laboratory of Water Engineering Materials and Structures, Nanning, Guangxi, 530023, China
}

\begin{abstract}
Soil erosion and socio-economic interacts with each other. Soil and water loss destroys land resources, causes non-point source pollution, affects the recycling and utilization of water resources, worsens the water environment, and even causes natural disasters such as collapse, landslides, and debris flows. Soil erosion affects regional socio-economic development. On the one hand, economic and social development has caused a sharp increase in people's demand for the development and utilization of natural resources, which has increased the occurrence of soil and water loss; on the other hand, economic and social development has promoted people's understanding of soil erosion and soil and water conservation, enabling people to consciously change their production and lifestyle, and having sufficient funds to invest in soil and water conservation and reduce soil erosion.
\end{abstract}

\section{Foreword}

Soil and water loss refers to various damages, movements, accumulation processes, and water loss caused by external forces such as water, wind, gravity, and freezing and thawing on the surface soil, parent material, and rocks. Water and soil loss in the narrow sense is specifically the water and soil loss caused by hydraulic erosion[1]. Soil and water loss is one of the major serious environmental problems in China currently[2]. There is a certain correlation between soil and water loss and social economy. On the one hand, soil and water loss will cause land productivity to decrease[3], and silted river banks[4], affecting the recycling and utilization of water resources, and affecting regional socio-economic development; on the other hand, socio-economic development will also have a certain impact on soil and water loss.

Since the twentieth century, the mutual influence and restrictive relationship between soil erosion and socio-economics have gradually been recognized. Many scholars have studied related content, especially in the past 10 years, the research on the correlation between soil erosion and socio-economics has been rapid development of. This article systematically summarizes the research on the correlation between soil and water loss and socioeconomics in China in recent years, and puts forward the development trend and research focus of future research on the correlation between soil and water loss and socioeconomics. The aim is to promote Chinese scholars to propose soil and water conservation measures and suggestions from a socioeconomic perspective, and to better promote the development of soil and water conservation in China.

\section{Research Methods of Soil and Water Loss and Socioeconomic Correlation}

The research methods for the correlation between soil erosion and socio-economics are mainly divided into two categories: qualitative research and quantitative research.

\subsection{Qualitative research}

In view of the wide variety of socio-economic factors and the wide range of aspects, some socio-economic factors are not easy to quantify, and the interaction between these factors is close. Until the beginning of the 21 st century, scholars mostly used qualitative analysis to study the relationship between soil erosion and socio-economics[5], mainly from the qualitative analysis of the impact of social and economic development on soil and water loss and the social and economic aspects of soil and water loss, put forward the social factors that cause soil and water loss[2], and discussed the importance of soil and water conservation to the sustainable development of society and economy.

\subsection{Quantitative research}

In recent years, as people gradually understand and grasp the basic conditions, internal meanings and connections

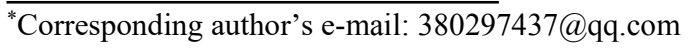


of various socioeconomic factors, and with the development of statistical theory, scholars' research on the relationship between soil erosion and socioeconomics has entered a stage of quantitative analysis. In terms of the social and economic impact of soil erosion, the opportunity cost method, shadow engineering method, market price method, restoration cost method, etc. have been applied to the monetary loss estimation of soil erosion, Cobb-Douglas Production Functional models have also been introduced into the socio-economic impact of soil erosion. In terms of socio-economic impacts on soil erosion, remote sensing, statistics, geostatistics, grey system theory, "stress-state-response" (PSR) system theory, and environmental Kuznets curve (EKC) theory are applied to society In the study of the impact of the economy on soil and water loss, scholars have used spatial processing software such as ArcGIS, ENVI, ERDAS to process some socio-economic information, and used statistical software such as GeoDa, spss. Analytical methods such as grey system analysis, introduced research models such as spherical model, linear regression model, spatial lag model, and spatial error model, explored the interference degree of human activities on soil erosion, and proposed the main index factors affecting soil erosion.

\section{Impact of Soil and Water Loss on Social Economy}

\subsection{Soil and water loss destroys land resources and reduces available land area}

The main types of hydraulic erosion are surface erosion and ditch erosion. The surface erosion washed away the fertile topsoil of cultivated land, garden land, and sparse forest land, which made the soil layer thin, the soil texture thickened, and the soil desertified. It even washed away the seeds or exposed the crop root system. The land yield was severely reduced, which greatly reduced land productivity and Land carrying capacity, gradually destroying land resources and affecting food security; the emergence of ditch erosion indicates that hydraulic erosion has developed to a more serious stage Gully erosion cuts the channel, interrupts traffic, and breaks the ground surface shape. The erosion ditch that runs through the slope and under the slope is a transport channel for runoff and sand production on slope farmland, which has increased the rate of soil erosion on the slope. In addition, the existence of erosion trenches prevents large agricultural machinery from passing through laterally, increasing the area of land destruction, reducing the efficiency of agricultural machinery, and reducing the available land area[6].

\subsection{Non-point source pollution caused by water and soil loss}

Soil and water loss is one of the main causes of non-point source pollution. Excessive reclamation, unreasonable production activities and land use methods lead to soil and water loss. The generated runoff carries a large amount of runoff and soil, and a large amount of nitrogen and phosphorus is used in agricultural production. Fertilizers such as potassium, potassium, pesticides, and heavy metals also flow into rivers, lakes, and reservoirs with runoff and soil, causing severe water surface source pollution[7], which not only causes loss of soil nutrients and soil fertility, but also causes eutrophication of water bodiesand deteriorate water quality.

\subsection{Water and soil loss affects the recycling and utilization of water resources}

Soil and water loss reduces soil conservation water resources and the ability of reservoirs and lakes to regulate and store water, which affects the recycling and utilization of water resources. On the one hand, the severe erosion of soil and water has taken away a large amount of surface fertile soil, the space for soil water storage, that is, the soil pores, has also gone, the soil water storage has decreased accordingly, the ability of the soil to conserve water sources has decreased, and surface runoff has increased; On the other hand, a large amount of sediment entrained by runoff caused by soil erosion is deposited along the terraces, ponds, reservoirs, lakes, rivers, etc. The deposition of terraced ponds reduces the service life of the project, raising the river bed causes the river channel to shrink, reducing Water storage facilities regulation function and natural river flood discharge capacity. The weakening of soil and various water conservancy projects' storage and storage functions has caused changes in the distribution of runoff during the year, making more rivers unable to release water during the flood season, less water impacting water use during non-flood seasons, and exacerbating the occurrence of droughts and floods[1], affecting the development of water conservancy projects and shipping.

\subsection{Soil and water loss deteriorates water environment and affects aquatic ecological security}

Severe soil and water loss has affected the circulation and utilization of water resources[8], making more water in the flood season and less water in the dry season; surface soil enters the water body in the form of sediment, and the sand content in the water body increases, increasing the turbidity of the water Soil and water loss causes siltation of reservoirs, lakes, rivers, etc., and at the same time, the amount of water in the dry season is reduced, which reduces the dilution and self-purification capacity of water bodies, reduces water environmental capacity, and accelerates water pollution. The above reasons have led to changes in the ecosystem environment of wetlands, reservoirs, rivers, lakes, etc., resulting in a decrease in species of animals and plants, a decrease in biodiversity, and affecting the safety of aquatic ecosystems. 


\subsection{Severe soil and water loss is likely to cause natural disasters}

Severe soil erosion can easily lead to natural disasters such as collapse, collapse mound, landslides, and debris flows. Soil and water loss is generally caused by the destruction of vegetation, leading to changes in runoff, soil and geological structures are affected, and geological disasters such as collapse and collapse mound are prone to occur. In the event of heavy rain, even serious natural disasters such as landslides and debris flows, these serious natural Disasters will not only destroy houses, roads, power communication facilities, etc., but also damage farmland, ponds, reservoirs and other water conservancy facilities. It will also severely disrupt river channels and affect shipping. It will also affect industrial and agricultural production in the river basin, urban safety, and people. The safety of life and property poses a huge threat[2].

\subsection{Soil and water loss affects social economy and exacerbates poverty}

Soil and water loss destroys land resources, affects the recycling and use of water resources, worsens the ecological environment, exacerbates natural disasters, endangers food, water supply, ecological security, and brings serious harm to the development of the national economy and the production and life of the people. Part of the development opportunities for improving the living conditions of agricultural production are lost, exacerbating poverty, especially in the vast karst areas. The unique geological hydrothermal conditions combined with the effects of soil and water erosion, are extremely prone to rocky desertification, making Local residents are trapped in rocky desertification poverty[9]. The study found that soil and water loss has a significant negative effect on agricultural production. The larger the share of soil and water loss and the deeper the degree of soil and water loss, the greater the negative impact on agricultural GDP. By estimating the monetary economic loss of soil erosion, it is found that the economic loss of soil erosion is huge, and the economic loss of nutrient loss caused by soil erosion is the largest.

\section{Social and economic impacts on soil erosion}

\subsection{Negative impact of socioeconomic on soil erosion}

Economic and social development has caused a sharp increase in people's demand for the development and utilization of natural resources, which has exacerbated the occurrence of soil and water loss. Socio-economic development factors are one of the important driving forces affecting soil and water loss. With the economic and social development, the population is growing too fast, the modernization process and the level of urbanization are constantly accelerating, which has led to a sharp increase in the demand for natural resources such as agriculture, forestry, animal husbandryand construction of infrastructure. People even for short-term benefits Destroying natural resources and the environment, causing a large number of new soil erosion. Soil and water loss is affected by five types of factors, including demographic, economic, technical, policy and institutional factors, and cultural factors. The proportion of slope farmland, population density, and forest land coverage are the three major socioeconomic factors that affect soil and water loss[4]; Some scholars have also found through research on autocorrelation that soil and water loss has agglomeration in spatial distribution, and the spatial variation of soil and water loss intensity on a small scale is mainly affected by random factors such as human activities[5], but not at the mesoscale. For example, studies have found that the distribution of soil and water loss areas and the distribution of the poor are geographically coupled. Poverty is often associated with excessively rapid population growth, low educational level of the population, and economic development which cause Man-made soil erosion. Weak awareness of soil and water conservation in the society, and insufficient funding due to poverty, which hinders the development of soil and water conservation[2].

\subsection{Positive impact of socioeconomic on soil erosion}

Economic and social development has promoted people's understanding of soil and water loss, and enabled people to consciously change their production and lifestyle, carry out water and soil conservation projects, and reduce soil and water loss.

On the one hand, with the social and economic development and the improvement of the people's material and cultural living standards, people's needs for the ecological environment have gradually increased, and the society's awareness of soil and water conservation has been increasing. People are gradually realizing the disadvantages of the traditional development model that used to pollute first and then treat, destruct first and then recover. It has become a social consensus to reduce resource consumption and damage to water and land resources. Soil and water conservation has become an important hotspot for all sectors of society, with a better atmosphere of public opinion and society. People can consciously change their production and lifestyle and reduce soil erosion.

On the other hand, the development of economy and society enables society to have surplus human and material resources to invest in soil and water conservation. The construction of terraces, tree planting, grass protection, and slope storage and drainage projects can not only increase soil infiltration, contain water storage, reduce soil erosion, but also adjust the structure of land use and agricultural industry, and promote efficient agriculture and ecological agriculture. The above measures promote the organic combination of peasants' income increase and rural development, achieve sustainable development of the rural economy, 
fundamentally solve the driving factors of soil erosion and prevent new soil erosion.

\section{Discussion}

Many scholars have used qualitative and quantitative research methods to conduct a large number of researches on the correlation between soil erosion and socioeconomics across the country. It is generally believed that soil erosion and socioeconomics affect each other and restrict each other.

On the one hand, soil and water loss damages land resources, causes non-point source pollution, affects the recycling and use of water resources, deteriorates the environment, and even causes natural disasters such as collapse, landslides, debris flows, and affects regional social and economical development. Economic and social development has increased people's demand for the development and utilization of natural resources, and exacerbated the occurrence of soil and water loss. On the other hand, economic and social development has promoted people's understanding of soil and water loss and soil and water conservation, enabling people to consciously change their production and lifestyle, investing human, material, and financial resources to carry out soil and water conservation and reduce soil and water loss.

However, the research on the correlation between soil erosion and socioeconomics is basically based on the independent research of scholars in a limited area. The similarities and differences between soil erosion and socioeconomic relationships in different regions of the country need to be further studied. From the perspective of socio-economics, regional measures and suggestions for soil and water conservation need to be further explored.

\section{References}

1. Lu Y.(2010) Harm of soil erosion and countermeasures. Gansu Agriculture, 283: 51-52.

2. Di B.F., Ning D.H., Lu S.L. (2006) Study on relationship between soil erosion and poverty. Bulletin of Soil and Water Conservation, 26(03):67-72.

3. Li B.Q., Fu J.X., Zhang S.Y.(2014) Harm of Soil and Water Loss and Prevention Countermeasures. Beijing Nongye, 2014 (24) :213-214.

4. WANG G., ZHANG Q.P., ZHENG H.J., YANG J., XIAO R.B. (2017) Temporal variation of soil and water loss and its social-economic driving forces in Jiangxi Province from 1987 to 2013. Ecological Science, 36(03):115-120.

5. Huang Q.F. (2015) Correlation analysis on soil erosion and socioeconomic development in Guizhou. Research of Soil and Water Conservation, 22(04):72-78.

6. Li H. (2016) Gully erosion evolvement and effect in typical black soil region. University of Chinese
Academy of Sciences, 2016.

7. Mo M.H., Fang S.W., Tu A.G. et al. Review of non-point source pollution from soil and water loss and its prevention and control. Soil and Water Conservation in China, 06:32-34.

8. Chang Y. (2012) The research of the harm of soil and water loss with the function of soil and water conservation. Northern Environmental, 24(02):161-163.

9. Su C.J., Yue T., Jiang L. et al. (2018) Correlation Research between Karst Rocky Desertification and Human Activities: A Case of Guangxi. Guangxi Sciences, 2018,25(6):709-714 